\title{
High prevalence of wild-type transthyretin deposition in patients with idiopathic carpal tunnel syndrome: a common cause of carpal tunnel syndrome in the elderly
}

Yoshiki Sekijima ${ }^{1,2}$, Shigeharu Uchiyama ${ }^{3}$, Kana Tojo ${ }^{1}$, Kenji Sano ${ }^{4}$, Yusaku Shimizu ${ }^{5}$,

Toshihiko Imaeda ${ }^{6}$, Yoshibonu Hoshii $^{7}$, Hiroyuki Kato ${ }^{3}$, Shu-ichi Ikeda ${ }^{1}$

${ }^{1}$ Department of Medicine (Neurology and Rheumatology), Shinshu University School of Medicine, 3-1-1 Asahi, Matsumoto 390-8621, Japan.

${ }^{2}$ Division of Clinical and Molecular Genetics, Shinshu University Hospital, 3-1-1 Asahi, Matsumoto 390-8621, Japan.

${ }^{3}$ Department of Orthopaedic Surgery, Shinshu University School of Medicine, 3-1-1 Asahi, Matsumoto 390-8621, Japan.

${ }^{4}$ Department of Laboratory Medicine, Shinshu University Hospital, 3-1-1 Asahi, Matsumoto 390-8621, Japan.

${ }^{5}$ Department of Neurology, Ina Central Hospital, 1313-1 Ina 396-8555, Japan

${ }^{6}$ Department of Food and Nutritional Environment, Kinjo Gakuin University, 2-1723 Ohmori, Moriyama-ku, Nagoya 463-8521, Japan,

${ }^{7}$ Department of Pathology, Yamaguchi University School of Medicine, Ube 755-8505, Japan

Correspondence: Yoshiki Sekijima, Department of Medicine (Neurology and Rheumatology), Shinshu University School of Medicine, 3-1-1 Asahi, Matsumoto 390-8621, Japan.

TEL +81-263-37-2673, FAX +81-263-37-3427, E-mail sekijima@,shinshu-u.ac.jp

Key words: carpal tunnel syndrome, transthyretin, amyloid, senile systemic amyloidosis, aging 
Funding: This study was supported by a Grant-in-aid for Scientific Research (20590695 to YS) and a grant form Amyloidosis Research Committee, the Ministry of Health, Labour and Welfare, Japan. The sponsors of the study had no role in study design, data collection, data analysis, data interpretation or writing of the report.

Conflicts of Interest: All authors have no conflicts of interest. 


\section{Summary}

Carpal tunnel syndrome is the most common type of entrapment neuropathy. However, the cause of carpal tunnel syndrome remains unclear in most cases. Senile systemic amyloidosis, induced by wild-type transthyretin deposition, is a prevalent aging-related disorder and often accompanied by carpal tunnel syndrome. In this study, we measured the frequency of unrecognized wild-type transthyretin deposition in idiopathic carpal tunnel syndrome patients. One-hundred and twenty-three patients with carpal tunnel syndrome, including 100 idiopathic patients, treated by carpal tunnel release surgery were analyzed. Tenosynovial tissues obtained at surgery were analyzed by Congo red and immunohistochemical staining. If staining for transthyretin was positive, the entire TTR gene was analyzed by direct DNA sequencing. We also analyzed tenosynovial tissues from 32 autopsy cases as controls. Thirty-four (34.0\%) patients with idiopathic carpal tunnel syndrome showed amyloid deposition in the tenosynovial tissue, and all amyloid showed specific immunolabelling with anti-transthyretin antibody. Direct DNA sequencing of the entire TTR gene did not reveal any mutations, indicating that all amyloid deposits were derived form wild-type transthyretin. Statistical analysis using logistic regression showed that the prevalence of transthyretin deposition in the idiopathic carpal tunnel syndrome group was significantly higher than that in controls (odds ratio $15.8,95 \%$ CI 3.3-75.7), and age and male gender were independent risk factors for transthyretin amyloid deposition. Our results demonstrate that wild-type transthyretin deposition is a common cause of carpal tunnel syndrome in elderly men. It is likely that many patients develop carpal tunnel syndrome as an initial symptom of senile systemic amyloidosis. 


\section{Introduction}

Carpal tunnel syndrome (CTS), the most common form of entrapment neuropathy, is estimated to occur in $3.8 \%$ of the general population [1], and its burden on society from lost productivity is substantial [2]. CTS accompanies various conditions and diseases, including pregnancy, obesity, diabetes, rheumatoid arthritis, sarcoidosis, purulent tenosynovitis, tuberculosis, systemic lupus erythematosus, hypo- or hyperthyroidism, gout and amyloidosis. However, its aetiology remains largely unclear in most patients and idiopathic CTS is the most common diagnosis [3]. Traditionally, idiopathic CTS was believed to be caused by an incompatibility between the size of the median nerve and the contents of the carpal tunnel, leading to increased pressure within the carpal tunnel and disturbance of blood flow to the median nerve [4]. However, recent magnetic resonance imaging (MRI), histological and biomechanical studies have strongly suggested that the development of idiopathic CTS is closely related to abnormalities of the synovial tissue within the carpal tunnel, although previously reported microscopic changes were nonspecific and inconclusive.

Senile systemic amyloidosis (SSA), induced by wild-type transthyretin (TTR) deposition [5], is a prevalent aging-related disorder, as about $25 \%$ of people over age 80 have TTR deposition in the heart [6], but it is usually detected by microscopic examination at autopsy. Although SSA is usually associated with cardiac disease, such as congestive heart failure and atrial fibrillation [7], TTR deposition is not limited to the heart and is found in systemic organs, including the aorta, lung, gastrointestinal tract, liver, kidney and connective tissues [6, 8]. CTS is one of the most common clinical manifestations of SSA and often precedes cardiac 
symptoms [9-11].

The prevalence of amyloid deposition in CTS patients has been investigated in a small number of studies [12-14]. However, the prevalence of wild-type TTR deposition in idiopathic CTS patients, as well as the clinical characteristics of CTS patients with wild-type TTR amyloid deposition remains unknown, since none of these studies analyzed the sequence of the TTR gene. Furthermore, the significance of TTR amyloid deposition for the onset of CTS is unclear due to the lack of controls. Here, we examined the frequency of wild-type TTR deposition in tenosynovial tissue in a cohort of 100 patients with idiopathic CTS by immunohistochemical staining with a highly specific and sensitive anti-TTR antibody and molecular diagnostic techniques.

\section{Materials and Methods}

\subsection{Patients}

From February 2008 to October 2010, 123 consecutive patients (31 male, 92 female) who were diagnosed with CTS and subsequently underwent carpal tunnel release surgery from three different clinical departments in Nagano Prefecture, Japan, were enrolled prospectively in this case-control study. We collected blood samples of and clinical information on these patients including body mass index (BMI). We also obtained tenosynovial tissues from CTS patients at carpal tunnel release surgery. This study was approved by the Ethical Committee of Shinshu University School of Medicine and written informed consent was obtained from each patient. CTS was diagnosed based on the patients' clinical history, physical examination, 
nerve conduction studies and MRI of the wrist. Physical examinations included the Tinel-like sign at the wrist, Phalen test and sensory disturbance detected over the median nerve-innervated area. To study only idiopathic CTS, we excluded the following conditions: pregnancy, rheumatoid arthritis, multiple myeloma, M-protein in the serum and urine, chronic renal failure under haemodialysis, diabetes mellitus, gout, gigantism, hypothyroidism, Colles' fracture, space-occupying lesions, chondrocalcinosis, osteoarthritis, tuberculous synovitis and previously diagnosed systemic amyloidosis, including familial amyloid polyneuropathy (FAP) and SSA. MRI was used to confirm that the patients did not have any space-occupying lesions or anatomical abnormalities, such as palmaris profundus or persistent median artery.

One-hundred of 123 patients were diagnosed with idiopathic CTS and analyzed in this study. The study population (idiopathic CTS patients) consisted of 26 men and 74 women ranging in age from 40 to 92 years (average age $67.3 \pm 12.1$ years). As controls, we also analyzed tenosynovial tissues from 32 autopsy cases (14 men and 18 women, average age $85.8 \pm 8.4$ years) without history of CTS. When synovial tissue was harvested from within the carpal tunnel, the median nerve configuration at the wrist was carefully observed to determine if there was any compressive deformity at the hook of the hamate level or enlargement of the median nerve at the proximal carpal tunnel. These deformations of the median nerve were typical findings of idiopathic CTS. None of the 32 autopsy cases had such findings. Since age and sex distribution were considerably different between CTS patients and controls, we employed logistic regression analysis to correct these differences (see 2.4. Statistical analysis). 


\subsection{Congo red and immunohistochemical analysis}

Tenosynovial tissues obtained at surgery were analyzed by Congo red and immunohistochemical staining. The degree of amyloidosis was divided into three categories and evaluated semiquantitatively $[12,13]$ : grades I, II and III had mild, moderate and severe amyloid deposition, respectively. Detailed definition of grading and clinical implications are summarised in Table 1.

Immunohistochemical analysis was performed as follows. After deparaffinisation, sections were treated with Peroxidase-Blocking Solution (Dako, Glostrup, Denmark) to inhibit endogenous peroxidase activity and with Protein Block, Serum-Free (Dako, Carpinteria, CA, USA) to inhibit nonspecific binding. Anti- $\lambda$ (118-134), anti-к (116-133) [15], anti-AA [16] and anti-TTR (115-124) [17] antibodies were applied to the sections as primary antibodies for $30 \mathrm{~min}$ at room temperature. The sections were then incubated with EnVision+ (Dako) as the secondary antibody for $30 \mathrm{~min}$ at room temperature. Immunoreactivity was visualized with $\mathrm{DAB}+($ Dako)

\subsection{Mass spectrometry and DNA analysis}

If staining for TTR was positive, matrix-assisted laser desorption ionization/time-of-flight (MALDI/TOF) mass spectrometric analysis of immunoprecipitated serum TTR molecules and direct DNA sequencing of entire TTR gene were performed to detect variant forms of TTR protein and TTR gene mutations. 


\subsection{Statistical analysis}

Statistical comparisons were performed between patients with idiopathic CTS and control subjects and between idiopathic CTS patients with and without TTR amyloid deposition. Logistic regression analysis was used to test the significance of TTR amyloid deposition for the onset of idiopathic CTS and to predict the independent risk factors for TTR amyloid deposition in idiopathic CTS patients. In logistic regression analysis between patients with idiopathic CTS and controls, the TTR amyloid deposition was set as a predictor (dependent variable), and age and sex were defined as independent factors or covariates. In the logistic regression analysis between idiopathic CTS patients with and without TTR amyloid deposition, one of three variables, age, sex or BMI, was set as a predictor and the other variables were defined as independent factors or covariates. We also compared demographic data of idiopathic CTS patients with TTR amyloid deposition to those without TTR amyloid deposition using the $\chi^{2}$ test (for binary outcomes) or Mann-Whitney $U$ test (for continuous variables). All statistical analyses were conducted using SPSS version 14.0J software (SPSS Inc., Chicago, IL, USA). The critical values for significance were set at $P<0.05$ or $95 \%$ confidence interval (CI) does not overlap 1.

\section{Results}

\subsection{Prevalence of TTR amyloid deposition in the idiopathic CTS patients}

Table 2 shows demographic and histopathological data for idiopathic CTS patients and 
control subjects. Thirty-four of $100(34.0 \%)$ patients with idiopathic CTS showed amyloid deposition in the tenosynovial tissue, and all amyloid deposits were specifically immunolabelled only with the anti-TTR antibody (Fig. 1). Semiquantitatively, TTR amyloid grade I was present in 9 cases (Fig. 2A, B), grade II in 10 cases (Fig. 2C, D) and grade III in 15 cases (Fig. 2E, F). On the other hand, only 7 (21.9\%) of the 32 individuals showed TTR amyloid deposition in the tenosynovial tissue in the control group and none of them had grade III amyloid deposition (Table 2). Statistical analysis by binomial logistic regression, corrected for age and sex, showed that the prevalence of TTR amyloid deposition in the idiopathic CTS group was significantly higher than that in the control group (odds ratio $15.8,95 \%$ CI 3.29 75.7, Table 3). Furthermore, the prevalence of TTR amyloid deposition grade II (moderate deposition) and grade III (severe deposition) in idiopathic CTS group were particularly high compared to that in the control group with an odds ratio of 18.5 and $1.50 \times 10^{9}$, respectively (Table 4), suggesting that considerable deposition of TTR amyloid was closely associated with the onset of CTS.

\subsection{Risk factors for TTR amyloid deposition}

In the idiopathic CTS group, the average age of patients with TTR amyloid deposition was $75.1 \pm 8.9$ years, whereas that of patients without TTR amyloid deposition was $63.3 \pm 11.5$ years (Table 5). The prevalence of TTR deposition separated by age was as follows: $0 / 6(0 \%)$ in the 40 - to 49 -year-old group, $3 / 24(12.5 \%)$ in the 50- to 59-year-old group, $6 / 23(26.1 \%)$ in the 60 - to 69 -year-old group, $14 / 29(48.3 \%)$ in the 70 - to 79 -year-old group, and 11/18 
(61.1\%) in those over 80 years old (Fig. 3A). Statistical analysis by logistic regression showed that age is an independent risk factor for TTR deposition and odds ratio per 1-year increase was 1.14 (95\% CI $1.06-1.23)$. The prevalence rates of TTR amyloid deposition in males and females were 17/26 (65.4\%) and 17/74 (23.0\%), respectively, as shown in Fig. 3B and 3C. The sex ratio of 17:17, the ratio of males to females in the idiopathic CTS with TTR amyloid deposition group, was significantly higher than that in the idiopathic CTS without TTR amyloid deposition group (9:57, Table 5). Statistical analysis by logistic regression showed that male gender was an independent risk factor for TTR amyloid deposition with an odds ratio of $11.0(95 \%$ CI $2.67-45.5)$. There were no statistically significant differences in the affected side or BMI between idiopathic CTS patients with and without TTR amyloid deposition (Table 5).

\subsection{Sequences of TTR protein and gene}

Serum TTR protein and the TTR gene were analyzed in 29 of the 34 idiopathic CTS patients with TTR amyloid deposition. Variant TTR protein and TTR gene mutation were not detected by MALDI/TOF mass spectrometry and DNA sequencing in each patient, indicating that all cases of amyloid deposition were derived form wild-type TTR. No idiopathic CTS patients with TTR amyloid deposition had symptoms or signs indicative of systemic amyloidosis including cardiomyopathy, or a family history of amyloidosis.

\section{Discussion}


The amyloidoses are a large group of postsecretory protein misfolding and deposition diseases.[18] There are over 20 secreted human proteins whose misfolding and misassembly outside the cell is linked to amyloidosis [19]. CTS could appear as an initial symptom in long-term haemodialysis-related ( $\beta_{2}$-microglobulin) amyloidosis, amyloid light chain (AL) amyloidosis, and TTR-related amyloidosis [12-14, 20, 21]. Misfolding of variant TTR induces the autosomal dominant genetic disorder, FAP [20-22]. while misfolding of wild-type TTR leads to the sporadic amyloid disease, SSA, which usually affects people over 80 years of age $[5,6]$.

The prevalence of amyloid deposition in CTS patients has been investigated in a small number of studies. Stein et al. [12] studied 140 biopsies from 108 CTS patients with various background diseases and found amyloid deposition in 23 patients (27 wrists) and TTR-related amyloid was present in 16 of these 27 cases. Nakamichi et al. [13] studied 135 biopsies from 108 idiopathic CTS patients and showed that 10 patients (15 wrists) had mild or moderate amyloid deposition in the tenosynovial tissues and TTR-related amyloid was present in 6 patients (9 wrists). On the other hand, Kyle et al. [14] performed immunohistochemical analyses of 35 CTS patients with local amyloid deposition and showed that 33 had tissue that reacted with TTR antiserum. These results suggest that TTR amyloid deposition may be a common cause of idiopathic CTS. However, the prevalence of wild-type TTR deposition in idiopathic CTS patients, as well as the clinical characteristics of CTS patients with TTR amyloid deposition remain unknown, due to patient selection bias, contamination of secondary CTS, and insufficient sensitivity and specificity of antibodies. In addition, neither 
the sequence of the TTR protein nor its gene has been reported. Furthermore, the significance of TTR amyloid deposition for the onset of CTS is unclear due to the lack of controls.

Unselected idiopathic CTS patients were enrolled in this study, and we analyzed the prevalence of wild-type TTR amyloid deposition by immunohistochemical staining with a highly sensitive and specific anti-TTR antibody and molecular diagnostic techniques. In the present study, the frequency of TTR amyloid deposition on tenosynovial tissues was $34.0 \%$ (65.4\% in males and $23.0 \%$ in females), which was much higher than previously reported frequencies of $11.4 \%(16 / 140)$ [12] and $6.7 \%(9 / 135)$ [13], respectively. This discrepancy is likely due to differences in study populations and sensitivity of anti-TTR antibodies. Most patients analyzed by Nakamichi et al. [13] were middle-aged women ( 5 men and 103 women; mean age, 56 years old), whose frequency of TTR amyloid deposition was relatively low in our study (Fig. 3C). In addition, their immunostaining results were ambiguous, as amyloid protein was not determined in many patients [13], suggesting insufficient sensitivity of antibodies. While all amyloid deposits reacted specifically with anti-TTR antibody in our study, Stein et al. [12] found many CTS patients with non-TTR amyloid deposition, as they enrolled patients with previously diagnosed systemic amyloidoses, including $\beta_{2}$-microglobulin amyloidosis, AL amyloidosis and AA amyloidosis. The findings of our case-control study showed that the prevalence of TTR deposition in the idiopathic CTS group was significantly higher than that in the controls (Table 3). Furthermore, the severity of amyloid deposition was closely associated with the onset of CTS (Table 4), suggesting that TTR amyloid deposition is a common cause of CTS, especially in elderly men. 
There are two likely hypotheses for the pathogenesis of wild-type TTR deposition in the tenosynovial tissue. The first is that TTR amyloid deposition is a result of mechanical wear and degeneration of the tendons and the surrounding synovium during everyday activities, which are believed to play important roles in the development of idiopathic CTS. It is also likely that TTR amyloid deposition facilitates degeneration of tenosynovial tissue, which then leads to a vicious circle. In fact, Kyle et al. [23] suggested that amyloid may be localized to the tenosynovium based on a retrospective review of 124 CTS patients with local amyloid deposition. Alternatively, TTR amyloid deposition on tenosynovial tissue may be part of systemic wild-type TTR amyloidosis, SSA, as all TTR amyloid is derived from circulating serum TTR. Actually, about half of the 10 patients with SSA previously diagnosed in our department developed CTS as an initial symptom [9-11]. Considering these findings and the observation that about $25 \%$ of people over age 80 have TTR deposition in the heart [6], it is reasonable to assume that most CTS patients with wild-type TTR deposition have a small amount of TTR amyloid deposition in systemic tissues, including the heart. However, it is likely to take time to accumulate sufficient amounts of amyloid to develop cardiac symptoms. Similarly, patients with wild-type TTR-related cardiomyopathy, SSA, may have TTR amyloid deposition in tenosynovial tissue, even when they do not have CTS symptoms.

In summary, wild-type TTR amyloid deposition is commonly observed in the synovial tissue inside the carpal tunnel in elderly male patients with idiopathic CTS. It is likely that many patients develop CTS as an initial symptom of SSA. Long-term follow-up of patients with wild-type TTR deposition in the tenosynovial tissue is necessary to elucidate the clinical 
picture of SSA. 


\section{Acknowledgments}

The authors thank Ms. E. Nomura for her technical support. This study was supported by a Grant-in-aid for Scientific Research (20590695 to YS) and a grant form Amyloidosis Research Committee, the Ministry of Health, Labour and Welfare, Japan. 


\section{References}

[1] Atroshi I, Gummesson C, Johnsson R, Ornstein E, Ranstam J, Rosen I. Prevalence of carpal tunnel syndrome in a general population. JAMA 1999; 282: 153-8.

[2] Foley M, Silverstein B, Polissar N. The economic burden of carpal tunnel syndrome: long-term earnings of CTS claimants in Washington State. Am J Ind Med 2007; 50: $155-72$.

[3] Uchiyama S, Itsubo T, Nakamura K, Kato H, Yasutomi T, Momose T. Current concepts of carpal tunnel syndrome: pathophysiology, treatment, and evaluation. J Orthop Sci 2010; 15: 1-13.

[4] Gelberman RH, Hergenroeder PT, Hargens AR, Lundborg GN, Akeson WH. The carpal tunnel syndrome. A study of carpal canal pressures. J Bone Joint Surg Am 1981; 63: $380-3$.

[5] Westermark P, Sletten K, Johansson B, Cornwell GG, III. Fibril in senile systemic amyloidosis is derived from normal transthyretin. Proc Natl Acad Sci U S A 1990; 87: 2843-5.

[6] Cornwell GG, III., Murdoch WL, Kyle RA, Westermark P, Pitkanen P. Frequency and distribution of senile cardiovascular amyloid. A clinicopathologic correlation. Am J Med 1983; 75: 618-23.

[7] Kyle RA, Spittell PC, Gertz MA, et al. The premortem recognition of systemic senile amyloidosis with cardiac involvement. Am J Med 1996; 101: 395-400.

[8] Pitkanen P, Westermark P, Cornwell GG, III. Senile systemic amyloidosis. Am J Pathol 
1984; 117: 391-9.

[9] Takei Y, Hattori T, Gono T, et al. Senile systemic amyloidosis presenting as bilateral carpal tunnel syndrome. Amyloid 2002; 9: 252-5.

[10] Takei Y, Hattori T, Tokuda T, et al. Senile systemic amyloidosis starting as bilateral carpal and left ulnar tunnel syndrome. Intern Med 2003; 42: 1050-1.

[11] Kodaira M, Sekijima Y, Tojo K, et al. Non-senile wild-type transthyretin systemic amyloidosis presenting as bilateral carpal tunnel syndrome. J Peripher Nerv Syst 2008; 13: $148-50$.

[12] Stein K, Storkel S, Linke RP, Goebel HH. Chemical heterogeneity of amyloid in the carpal tunnel syndrome. Virchows Arch A Pathol Anat Histopathol 1987; 412: 37-45.

[13] Nakamichi KI, Tachibana S. Amyloid deposition in the synovium and ligament in idiopathic carpal tunnel syndrome. Muscle Nerve 1996; 19: 1349-51.

[14] Kyle RA, Gertz MA, Linke RP. Amyloid localized to tenosynovium at carpal tunnel release. Immunohistochemical identification of amyloid type. Am J Clin Pathol 1992; 97: 250-3.

[15] Hoshii Y, Setoguchi M, Iwata T, et al. Useful polyclonal antibodies against synthetic peptides corresponding to immunoglobulin light chain constant region for immunohistochemical detection of immunoglobulin light chain amyloidosis. Pathol Int $2001 ; 51: 264-70$.

[16] Imada N. Pathological study on amyloidosis. Isolation and purification of amyloid fibril protein and biochemical and immunological analysis. Yamaguchi Med J 1981; 30: 
149-62.

[17] Gustavsson A, Engstrom U, Westermark P. Mechanisms of transthyretin amyloidogenesis. Antigenic mapping of transthyretin purified from plasma and amyloid fibrils and within in situ tissue localizations. Am J Pathol 1994; 144: 1301-11.

[18] Dobson CM. Protein folding and misfolding. Nature 2003; 426: 884-90.

[19] Westermark P, Benson MD, Buxbaum JN, et al. A primer of amyloid nomenclature. Amyloid 2007; 14: 179-83.

[20] Wallace MR, Conneally PM, Benson MD. A DNA test for Indiana/Swiss hereditary amyloidosis (FAP II). Am J Hum Genet 1988; 43: 182-7.

[21] Connors LH, Lim A, Prokaeva T, Roskens VA, Costello CE. Tabulation of human transthyretin (TTR) variants, 2003. Amyloid 2003; 10: 160-84.

[22] Sekijima Y, Wiseman RL, Matteson J, et al. The biological and chemical basis for tissue-selective amyloid disease. Cell 2005; 121: 73-85.

[23] Kyle RA, Eilers SG, Linscheid RL, Gaffey TA. Amyloid localized to tenosynovium at carpal tunnel release. Natural history of 124 cases. Am J Clin Pathol 1989; 91: 393-7. 


\section{Figure Legends}

Fig. 1 Histopathological findings of tenosynovial tissue from the carpal tunnel. Congo red staining showed amyloid deposits in the connective tissues (A; B, under polarized light) and all these deposits were specifically immunolabelled with an anti-TTR antibody (C-F; Immunohistochemical staining with anti-TTR (C), anti- $\kappa(D)$, Anti- $\lambda(E)$ and anti-AA (F) antibody). A 77 year-old woman, grade II.

Fig. 2 TTR amyloid deposition on tenosynovial tissue. Top row (A, C, E) shows Congo red staining. Bottom row (B, D, F) shows immunohistochemical staining with anti-TTR antibody. (A, B) A 76-year-old man, grade I. (C, D) A 66-year-old woman, grade II. (E, F) an 81-year-old man, grade III.

Fig. 3 Prevalence of TTR deposition in idiopathic CTS patients separated by age and sex. (A) All idiopathic CTS patients $(n=100)$, (B) male idiopathic CTS patients $(n=26)$ and $(\mathrm{C})$ female idiopathic CTS patients $(n=74)$. Data are expressed as relative percentages, with the number of patients TTR-positive and number of patients tested in each age group indicated above each bar. Statistical analysis by logistic regression showed that age (odds ratio per 1-year increase 1.14, 95\% CI 1.06-1.23) and male gender (odds ratio 11.0, 95\% CI 2.6745.5) are independent risk factors for TTR deposition. 
Table 1. Definition of amyloid deposition grades and clinical implications.

\begin{tabular}{|c|l|l|}
\hline Grade & \multicolumn{1}{|c|}{ Definition } & \multicolumn{1}{|c|}{ Clinical implications } \\
\hline $\begin{array}{c}\text { I } \\
\text { (mild) }\end{array}$ & $\begin{array}{l}\text { Small amount of TTR amyloid deposition ( } \phi \\
<100 \mu \mathrm{m}) \text { in some parts of biopsied tissue }\end{array}$ & $\begin{array}{l}\text { Probably not related to the onset } \\
\text { of CTS }\end{array}$ \\
\hline $\begin{array}{c}\text { II } \\
\text { (moderate) }\end{array}$ & $\begin{array}{l}\text { Considerable deposition of TTR amyloid }(\phi \geq \\
100 \mu \mathrm{m}) \text { in many parts of biopsied tissue }\end{array}$ & $\begin{array}{l}\text { Probably related to the onset of } \\
\text { CTS }\end{array}$ \\
\hline $\begin{array}{c}\text { III } \\
\text { (severe) }\end{array}$ & $\begin{array}{l}\text { Massive deposition of TTR amyloid in most } \\
\text { parts of biopsied tissue }\end{array}$ & Probably direct cause of CTS \\
\hline
\end{tabular}


Table 2. Prevalence of TTR amyloid deposition in idiopathic CTS patients and controls (autopsy cases)

\begin{tabular}{|c|c|c|c|c|c|c|}
\hline & & & \multicolumn{2}{|c|}{ Idiopathic CTS group } & \multicolumn{2}{|c|}{$\begin{array}{c}\text { Control group } \\
\text { (autopsied patients) }\end{array}$} \\
\hline \multicolumn{3}{|c|}{ Number of individuals } & \multicolumn{2}{|c|}{100} & \multicolumn{2}{|c|}{32} \\
\hline \multicolumn{3}{|c|}{ Age (mean \pm SD) } & \multicolumn{2}{|c|}{$67.3 \pm 12.1$} & \multicolumn{2}{|c|}{$85.8 \pm 8.4$} \\
\hline \multicolumn{3}{|c|}{ Sex (male : female) } & \multicolumn{2}{|c|}{ 26:74 } & \multicolumn{2}{|c|}{$14: 18$} \\
\hline \multirow{5}{*}{$\begin{array}{c}\text { TTR } \\
\text { amyloid } \\
\text { deposition }\end{array}$} & \multicolumn{2}{|c|}{-} & $66 / 100$ & (66.0\%) & $25 / 32$ & $(78.1 \%)$ \\
\hline & \multicolumn{2}{|c|}{+} & $34 / 100$ & $(34.0 \%)$ & $7 / 32$ & $(21.9 \%)$ \\
\hline & \multirow{3}{*}{ grade } & I & $9 / 100$ & $(9.0 \%)$ & $4 / 32$ & $(12.5 \%)$ \\
\hline & & II & $10 / 100$ & $(10.0 \%)$ & $3 / 32$ & $(9.4 \%)$ \\
\hline & & III & $15 / 100$ & $(15.0 \%)$ & $0 / 32$ & $(0 \%)$ \\
\hline
\end{tabular}


Table 3. Logistic regression analysis between idiopathic CTS patients and controls

\begin{tabular}{|c|c|c|c|c|c|c|c|}
\hline \multirow[b]{2}{*}{ Parameter } & \multirow[b]{2}{*}{ B } & \multirow[b]{2}{*}{ SE B } & \multirow[b]{2}{*}{ Wald } & \multirow[b]{2}{*}{$P$} & \multirow{2}{*}{$\begin{array}{c}\text { Exp (B) } \\
\text { (Odds ratio) }\end{array}$} & \multicolumn{2}{|c|}{ 95\% CI of $\operatorname{Exp}(B)$} \\
\hline & & & & & & Lower & Upper \\
\hline Age & -0.246 & 0.051 & 23.389 & $<0.001$ & 0.782 & 0.708 & 0.864 \\
\hline Sex & 1.757 & 0.761 & 5.335 & 0.021 & 5.793 & 1.305 & 25.721 \\
\hline $\begin{array}{l}\text { TTR amyloid } \\
\text { deposition }\end{array}$ & 2.759 & 0.800 & 11.907 & 0.001 & 15.788 & 3.294 & 75.684 \\
\hline Constant & 18.173 & 3.764 & & & & & \\
\hline
\end{tabular}


Table 4. Logistic regression analysis between idiopathic CTS patients and controls separated by TTR amyloid deposition grade

\begin{tabular}{|c|c|c|c|c|c|c|c|}
\hline \multirow[b]{2}{*}{ Parameter } & \multirow[b]{2}{*}{ B } & \multirow[b]{2}{*}{ SE B } & \multirow[b]{2}{*}{ Wald } & \multirow[b]{2}{*}{$P$} & \multirow{2}{*}{$\begin{array}{c}\operatorname{Exp}(\mathrm{B}) \\
\text { (Odds ratio) }\end{array}$} & \multicolumn{2}{|c|}{$95 \%$ CI of $\operatorname{Exp}(B)$} \\
\hline & & & & & & Lower & Upper \\
\hline Age & -0.237 & 0.051 & 21.729 & $<0.001$ & 0.789 & 0.714 & 0.871 \\
\hline Sex & 1.896 & 0.773 & 6.010 & 0.014 & 6.659 & 1.462 & 30.321 \\
\hline $\begin{array}{l}\text { TTR amyloid } \\
\text { deposition }\end{array}$ & & & 8.460 & 0.037 & & & \\
\hline Grade I & 2.105 & 0.907 & 5.393 & 0.020 & 8.210 & 1.389 & 48.531 \\
\hline Grade II & 2.917 & 1.180 & 6.106 & 0.013 & 18.486 & 1.828 & 186.925 \\
\hline Grade III & 21.130 & 9,330 & 0.000 & 0.998 & $1.502 \times 10^{9}$ & & \\
\hline Constant & 17.423 & 3.767 & & & & & \\
\hline
\end{tabular}

$\mathrm{R}^{2}=0.69$ (Nagelkerke), Model: $\chi^{2}(3)=19.7, \quad \mathrm{P}<0.001$

$B$, unstandardized coefficient; SE B, standard error of B 
Table 5. Characteristics of patients with idiopathic CTS with and without TTR amyloid deposition

\begin{tabular}{|l|c|c|c|}
\hline & $\begin{array}{c}\text { Idiopathic CTS with } \\
\text { TTR amyloid deposition }\end{array}$ & $\begin{array}{c}\text { Idiopathic CTS without } \\
\text { TTR amyloid deposition }\end{array}$ & $P$ \\
\hline Age (mean \pm SD) & $75.1 \pm 8.9$ & $63.3 \pm 11.5$ & $2.6 \times 10^{-6} *$ \\
\hline Sex (male $:$ female) & $17: 17$ & $9: 57$ & $8.6 \times 10^{-6} * *$ \\
\hline BMI (mean \pm SD) & $23.8 \pm 3.7$ & $23.4 \pm 5.0$ & $0.41 *$ \\
\hline Location of CTS (right $:$ left) & $26: 8$ & $39: 27$ & $0.08 * *$ \\
\hline
\end{tabular}

$P$-values were obtained using *Mann-Whitney $U$ test (for continuous variables) or $* * \chi^{2}$ test (for binary outcomes). 


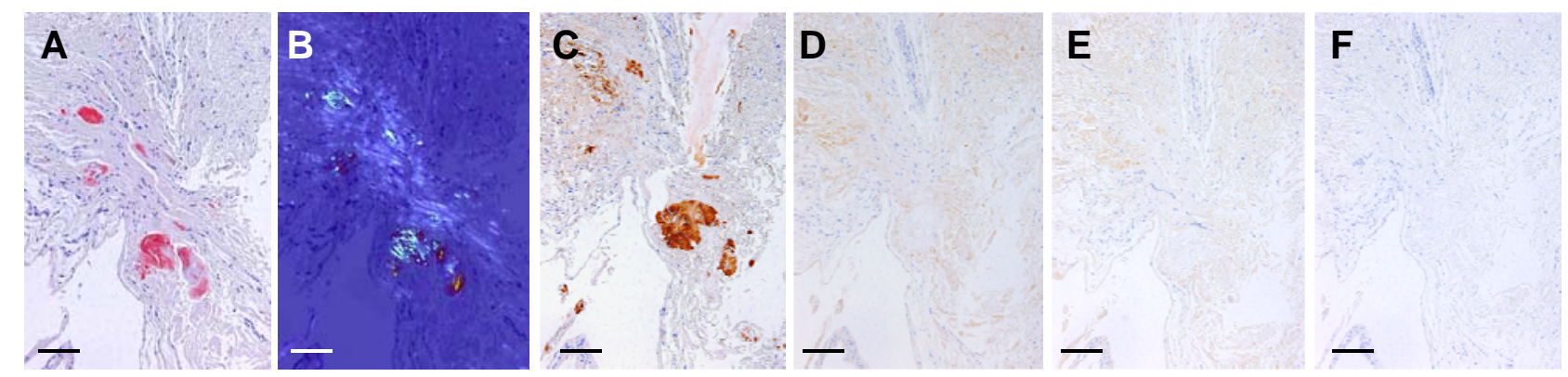

Figure 1

Sekijima et al. 


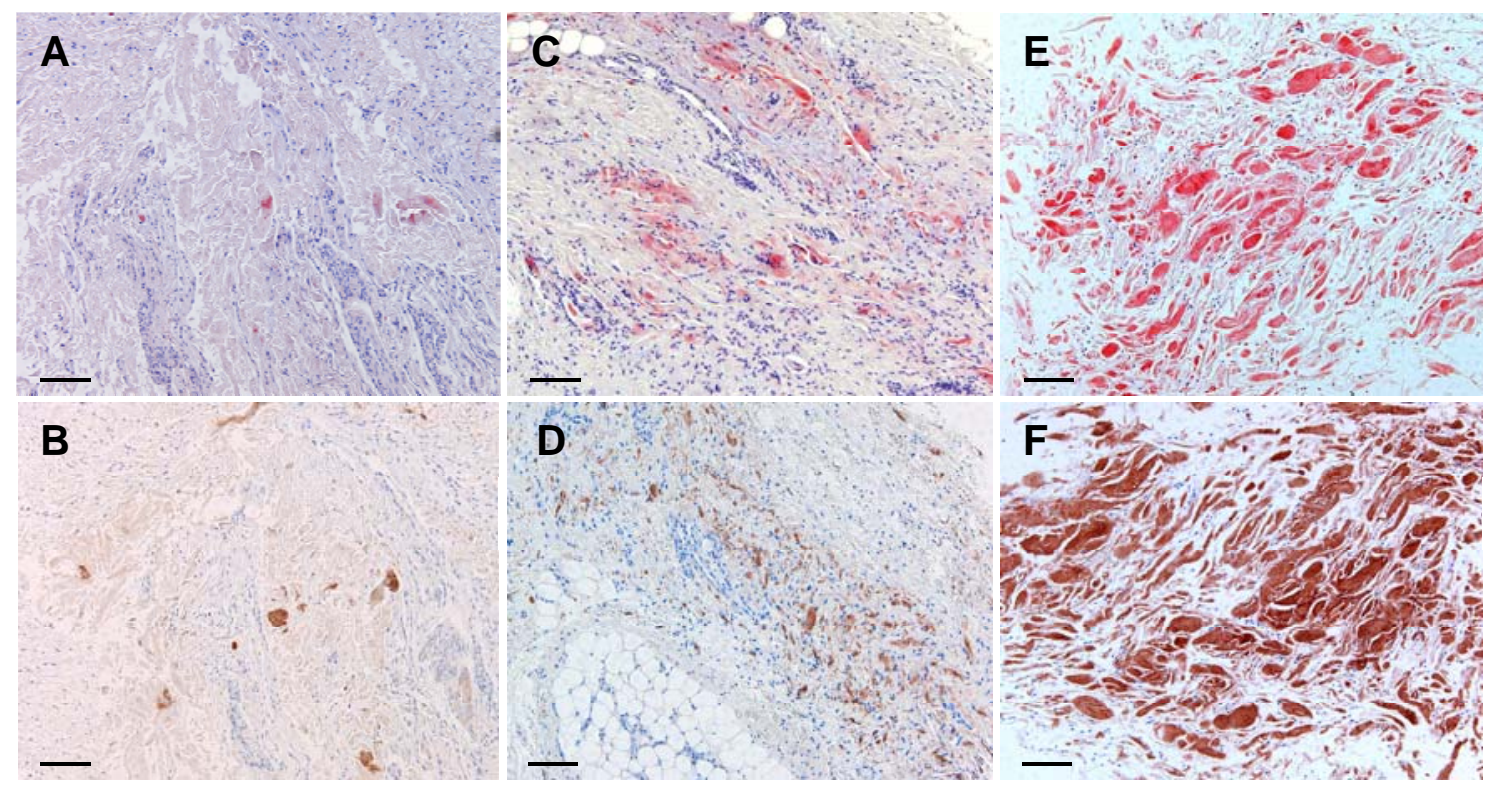

Figure 2

Sekijima et al. 
A. All patients

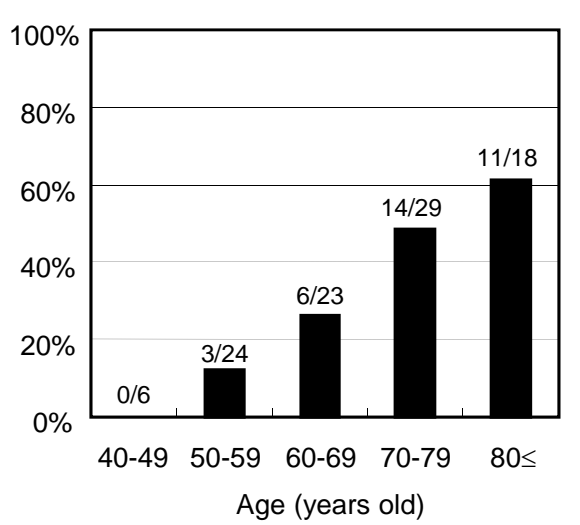

B. Male patients

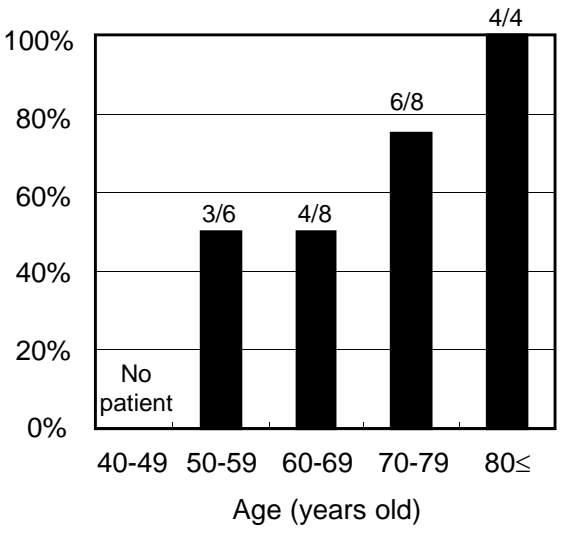

C. Female patients

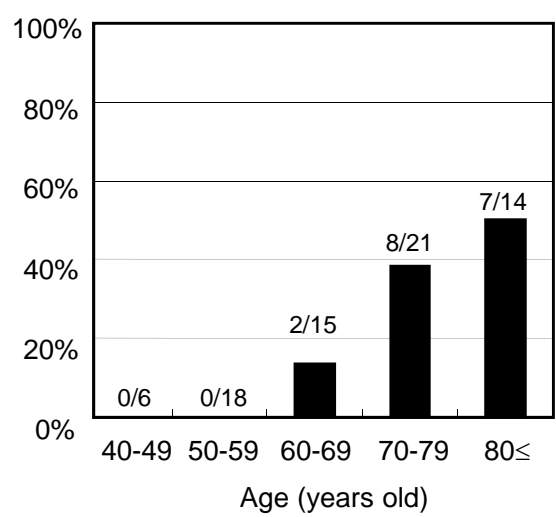

Figure 3

Sekijima et al. 